\title{
Tumor Results Domain
}

National Cancer Institute

\section{Source}

National Cancer Institute. Tumor Results Domain. NCI Thesaurus. Code C106578.

A findings domain that represents quantitative measurements and/or qualitative assessments of the tumors identified in the TU domain. 